\title{
Estudio Morfométrico de los Bronquios Principales y del Bronquio Intermedio en una Muestra Cadavérica Colombiana
}

\author{
Morphometric Study of the Main Bronchi and the \\ Intermediate Bronchus in a Colombian Cadaveric Sample
}

Luis Gabriel Parra-Lara ${ }^{1,2}$; Luz Marina Corrales ${ }^{3,5}$; Janneth Zúñiga ${ }^{4}$ \& Gloria Patricia Baena-Caldas ${ }^{4,5}$

PARRA-laRA, L. G.; CORRALES, L. M.; ZÚÑIGA, J. \& BAENA-CALDAS, G. P. Estudio morfométrico de los bronquios principales y del bronquio intermedio en una muestra cadavérica colombiana. Int. J. Morphol., 37(4):1252-1257, 2019.

RESUMEN: Conocer la morfometría en longitud, circunferencia y angulación de los bronquios principales y del bronquio intermedio pueden ser datos relevantes en procedimientos radiológicos, quirúrgicos e inclusive en eventos que amenazan la vida como la broncoaspiración. Existe poca evidencia acerca de la morfometría de los bronquios principales y del bronquio intermedio, es por ello que el presente estudio tuvo como objetivo describir la morfometría de los bronquios principales y del bronquio intermedio en una muestra de población cadavérica colombiana. Se realizó un estudio descriptivo donde se seleccionaron 29 especímenes de Cali, Colombia; 11 correspondieron a cadáveres y 18 a órganos aislados del tracto respiratorio inferior; se incluyeron los cadáveres con las estructuras debidamente disecadas y se excluyeron las muestras con estructuras anatómicas ausentes o dañadas y que afectaran la realización de las mediciones. La media de longitud del bronquio principal derecho fue de $1,69 \mathrm{~cm}$ IC $95 \%(1,51-1,87)$ la media de la longitud del bronquio intermedio fue de IC $95 \% 2,89 \mathrm{~cm}(2,66$ - 3,12), la media de longitud del bronquio principal izquierdo fue de 3,34 cm IC 95\% (3,01 - 3,68); el ángulo interbronquial tiene una media de $80,17^{\circ}$ IC $95 \%(72,87-87,48)$. Las mediciones del bronquio principal derecho concuerdan con las reportadas en la literatura, lo contrario ocurre con el bronquio principal izquierdo; la medición del ángulo interbronquial muestra diferencias a la reportada por la literatura. Este estudio muestra que a pesar de que existen nomenclaturas y consensos internacionales, estos no han sido implementados correctamente por los profesionales de la salud y el término de bronquio intermedio es utilizado en la práctica diaria, por lo que se hace necesario que las Sociedades de Anatomía y Morfología vuelvan a discutir su importancia y reconocimiento en la nomenclatura.

PALABRAS CLAVE: Anatomía; Bronquios; Cadáver.

\section{INTRODUCCIÓN}

Los pulmones y el árbol bronquial constituyen la vía respiratoria inferior del ser humano. Anatómicamente, el árbol bronquial inicia en la bifurcación de la tráquea, a nivel de la carina que se encuentra a la altura del ángulo esternal, donde se originan los bronquios principales derecho (BPD) e izquierdo (BPI). Estas son estructuras anatómicas tubulares que se ramifican en bronquios lobares seguidos de los bronquios segmentales, que a su vez se distribuyen en un determinado sector del parenquima pulmonar denominado segmento broncopulmonar (Delgado García, 1996).

El BPD es más corto, grueso y vertical que el BPI (Minnich \& Mathisen, 2007), conservando una orientación muy similar a la de la tráquea, lo que explica el por qué suele ser el sitio donde se alojan los cuerpos extraños broncoaspirados, siendo este evento clínico más frecuente en los niños menores de cinco años ( $84 \%$ de los casos) (AlvaradoLeón et al., 2011). El porcentaje de broncoaspiraciones ubicadas en el BPD corresponde a 52,5\% en niños y $69 \%$ en adultos (Baharloo et al., 1999).

El BPD emite inicialmente el bronquio lobar superior (BLS) y se continúa como bronquio intermedio (BI), el cual da origen, a su vez, a los bronquios lobares medio (BLM) e inferior (BLI). Por su parte, el BPI, más delgado, largo y oblicuo, da origen solamente a los BLS y BLI (Minnich \& Mathisen). Cabe resaltar que son escasos los estudios morfométricos que soportan información sobre la longitud y circunferencia de los bronquios, así como la angulación que estos presentan con respecto a la tráquea (Almaguer Rodríguez et al., 2001).

\footnotetext{
${ }^{1}$ Escuela de Medicina, Facultad de Salud, Universidad del Valle, Cali, Colombia.

${ }^{2}$ Centro de Investigaciones Clínicas (CIC), Fundación Valle del Lili, Cra. 98 \# 18-49, Cali 760032, Colombia.

${ }^{3}$ Facultad de Salud, Universidad Santiago de Cali, Cali, Colombia.

${ }^{4}$ Departamento de Morfología, Escuela de Ciencias Básicas, Facultad de Salud, Universidad del Valle, Cali, Colombia.

${ }^{5}$ Universidad Libre, sede Cali, Colombia.
} 
El patólogo William Ewart (1848-1929) conocido como el padre de la anatomía segmental, fue el primero en describir el BI en el libro "The bronchi and pulmonary blood-vessels", al cual llamó "intermediate stem" haciendo referencia a su ubicación anatómica intermedia entre los BLS y BLM (Ewart, 1889). Posteriormente, otros investigadores como Brock (1946) y Jackson \& Huber (1943) continuaron trabajando en la descripción del árbol bronquial, siendo estos últimos quienes le dieron un nombre definitivo a cada segmento, haciendo un gran aporte al desarrollo de la broncoscopia y a la nomenclatura anatómica actual. Después de la clasificación de Jackson \& Huber, aparecieron otras nomenclaturas para la segmentación bronquial como la de Boyden (1953), Ikeda (1974) y Cortese \& Prakash (1994); sin embargo, ninguna de estas clasificaciones tiene en cuenta la definición de BI o "intermediate stem" realizada por Ewart.

Actualmente, la nomenclatura aceptada por la Nómina Anatómica y por las diferentes asociaciones médicas sobre la anatomía broncopulmonar está basada en el consenso de la Thoracic Society, reunida en 1949, la cual acordó que lo que Ewart denominó BI es parte del BPD y se aprobó por unanimidad que la parte del BPD que va desde la carina hasta el margen inferior del BLS, debe ser llamado "superior" y que la parte que va desde este margen hasta el margen superior del bronquio segmental superior del BLI debe llamarse "inferior"; es decir, que lo que corresponde al BI debe llamarse "parte inferior del BPD” (Thoracic Society, 1950; Comité Federal sobre Terminología Anatómica, International Federation of Associations of Anatomists \& Sociedad Anatómica Española, 2001). Es de destacar que, a pesar del consenso de la Thoracic Society y de la nomenclatura aceptada por la Nómina Anatómica, todavía hay investigadores y profesionales de la salud que siguen empleando el término de BI para referirse a la parte inferior del BPD (Minnich \& Mathisen; Sebbagh et al., 2012; García Araque et al., 2014).

Dado que no se encontraron reportes en la literatura revisada con respecto a estudios morfométricos de estos bronquios en la población colombiana, se tuvo como objetivo hacerlo en una muestra cadavérica y describirla.

\section{MATERIAL Y MÉTODO}

Se realizó un estudio descriptivo con muestreo aleatorio simple, de especímenes cadavéricos previamente fijados en una solución de formaldehído al $5 \%$, pertenecientes a las salas de disección de una universidad de Cali,
Colombia. Se tuvo como criterio de inclusión que las estructuras a estudiar estuvieran debidamente disecadas y como criterio de exclusión, que las estructuras anatómicas del tracto respiratorio inferior estuvieran ausentes o dañadas y que afectaran la realización de las mediciones.

Se disecó el árbol bronquial de los cadáveres, bilateralmente, y de los pulmones aislados, hasta los bronquios lobares (Fig. 1A). Posteriormente, se midió tanto la longitud como la circunferencia/calibre de los bronquios principales y del BI, empleando una cinta métrica (marca "Davinci Measurment System"), con la que se obtuvieron los datos en centímetros. Para medir los ángulos se utilizó un transportador de ángulos en grados sexagesimales. Las mediciones fueron realizadas en diferentes sesiones, en un tiempo de 4 semanas; se realizaron pruebas interobservador para todas las mediciones.

Las medidas de la longitud de los bronquios y sus circunferencias se determinaron de la siguiente forma: BPD. Su longitud fue medida en su cara anterior, iniciando en la bifurcación de la tráquea hasta el margen inferior del BLS. Mientras que la circunferencia se midió a nivel de su origen; BI. Su longitud se midió iniciando en el margen inferior del BLS derecho hasta el margen superior del BLM, pasando por la cara anterior del BI. Su circunferencia se midió a nivel del margen inferior del origen del BLS derecho; BPI. Se midió su longitud por su cara anterior desde la bifurcación de la tráquea hasta el origen del BLI izquierdo. La circunferencia se midió a nivel del origen del BPI (Fig. 1B).

Los ángulos fueron medidos así: Ángulo interbronquial $(\alpha)$. Se tomó como vértice en el margen inferior de la bifurcación de la tráquea, por su cara externa, con semirrectas de los márgenes inferiores del BPI y BPD; Ángulo del BPD ( $\beta)$. Ángulo con vértice en el origen del BPD, a nivel de su margen superior, con semirrectas definidas por el margen lateral derecho de la tráquea y el margen superior del BPD; Ángulo del BPI ( $\theta)$. Ángulo con vértice en el origen del BPI, a nivel de su margen superior, con semirrectas definidas por el margen lateral izquierdo de la tráquea y el margen superior del BPI (Fig. 1C).

Las variables numéricas se resumieron usando medidas de tendencia central (promedio o mediana) y medidas de dispersión (desviación estándar y rango intercuartílico), según la prueba de Shapiro-Wilk. Se realizó cálculo de sus intervalos de confianza, con un nivel de significancia estadística de 0,05, utilizando la distribución t-student. Los análisis estadísticos se realizaron en STATA ${ }^{\circledR}$ (StataCorp, 2014, Stata 14 Base Reference Manual, College Station, TX: StataPress). 


\section{RESULTADOS}

Se seleccionaron 29 especímenes, de los cuales 11 correspondieron a cadáveres completos (todos de sexo masculino) y 18 a órganos aislados del tracto respiratorio inferior. Las mediciones realizadas en el anfiteatro se resumen en la Tabla I.

La media de la longitud del BPD fue de 1,69 cm IC $95 \%(1,51-1,87)$ y la media de su circunferencia en el origen 5,28 cm IC $95 \%(4,87-5,7)$. La media de la longitud del BI fue de $2,89 \mathrm{~cm}$ IC $95 \%(2,66-3,12)$ y la media de su circunferencia en el origen 3,97 cm IC $95 \%(3,71-$ 4,22). La media de longitud del BPI fue de $3,34 \mathrm{~cm}$ IC 95 $\%(3,01-3,68)$ y la media de su circunferencia $4,49 \mathrm{~cm}$ IC $95 \%(4,23-4,75)$.

Respecto a los ángulos, él ángulo del BPD $(\beta)$ respecto a la tráquea fue de $136,55^{\circ}$ IC $95 \%(130,63-142,47)$ y el ángulo del BPI $(\theta)$ respecto a la tráquea fue de $134,48^{\circ}$ IC $95 \%(128,23-140,74)$. El ángulo interbronquial $(\alpha)$ presentó una media de $80,17^{\circ}$ IC $95 \%(72,87-87,48)$.

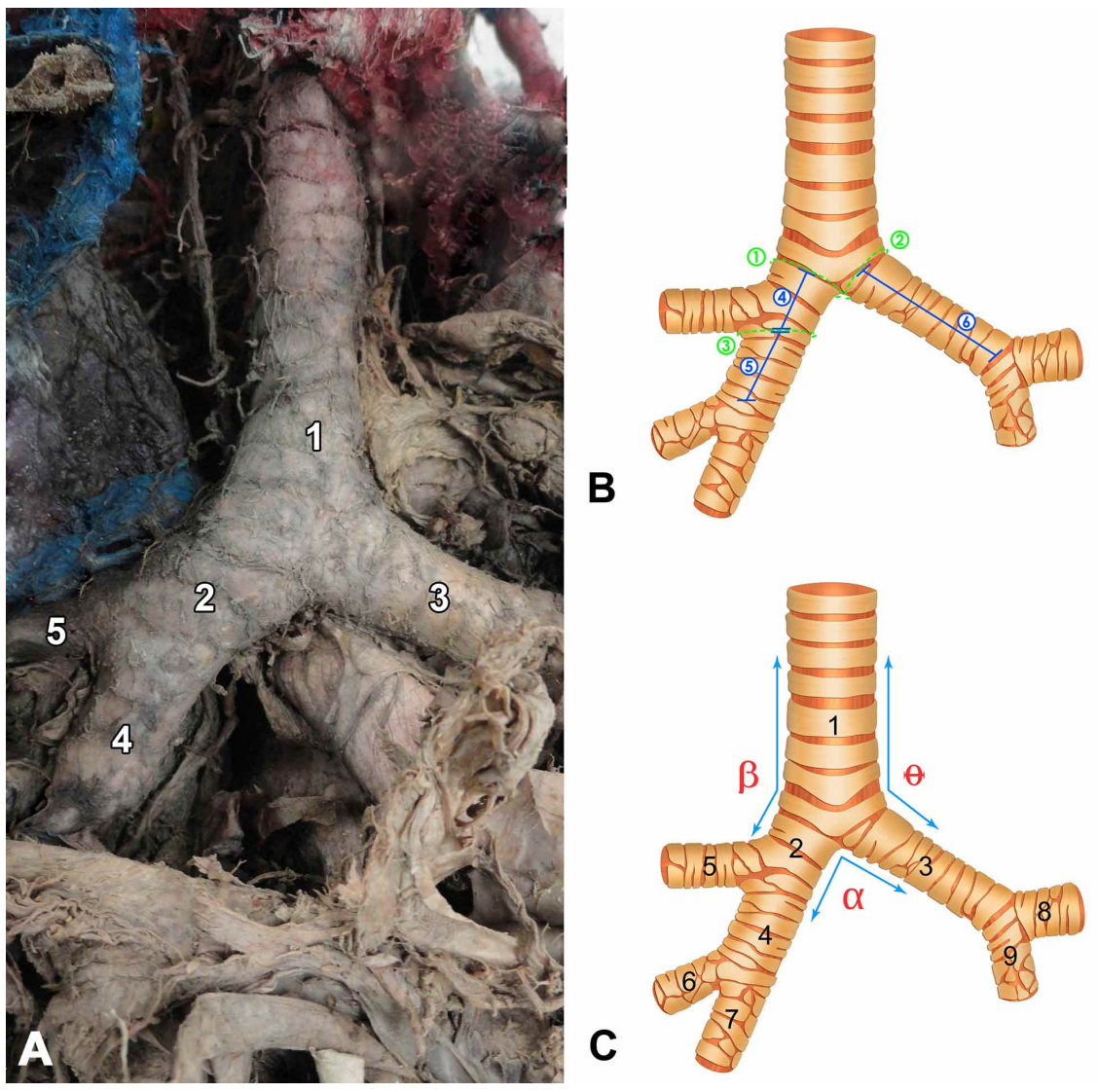

Fig. 1. Las figuras corresponden a una vista frontal del árbol traqueobronquial, donde se observa la tráquea (1) que se bifurca en el bronquio principal derecho (BPD) (2) y en el bronquio principal izquierdo (BPI) (3); en el lado derecho se observa al bronquio intermedio (BI) (4) iniciando a nivel del margen inferior del bronquio lobar superior (5). La Fig. 1A corresponde a un árbol traqueobronquial de cadáver in situ que fue estudiado. La Fig. 1B muestra él sitio donde se realizaron las mediciones de la circunferencia del BPD, BPI y BI (líneas discontinuas en verde $1,2,3$, respectivamente), y la manera como se midió la longitud de los BPD, BI y BPI (líneas continuas en azul 4, 5 y 6 , respectivamente). La Fig. 1C evidencia la ubicación del ángulo interbronquial $(\alpha)$, del ángulo del $\operatorname{BPD}(\beta)$ y del ángulo del BPI $(\theta)$.

Tabla I. Mediciones de la longitud, circunferencia y ángulos de los bronquios $(n=29)$.

\begin{tabular}{llcccccc}
\hline Mediciones & & Media & DE & EE & \multicolumn{2}{c}{ Intervalo confianza 95\% } & Prueba Shapiro \\
\hline \multirow{3}{*}{ Longitudes } & BPD & 1,69 & 0,47 & 0,09 & 1,51 & 1,87 & 0,97 \\
& BI & 2,89 & 0,62 & 0,11 & 2,66 & 3,12 & 0,07 \\
& BPI & 3,34 & 0,88 & 0,16 & 3,01 & 3,68 & 0,64 \\
Circunferencia & BPD & 5,28 & 1,09 & 0,20 & 4,87 & 5,70 & 0,06 \\
& BI & 3,97 & 0,68 & 0,13 & 3,71 & 4,22 & 0,92 \\
& BPI & 4,49 & 0,69 & 0,13 & 4,23 & 4,75 & 0,28 \\
Ángulos & BPD $(\beta)$ & 136,55 & 15,57 & 2,89 & 130,63 & 142,47 & 0,90 \\
& BPI $(\theta)$ & 134,48 & 16,45 & 3,05 & 128,23 & 140,74 & 0,33 \\
& Interbronquial $(\alpha)$ & 80,17 & 19,21 & 3,57 & 72,87 & 87,48 & 0,70 \\
\hline
\end{tabular}

BPD: bronquio principal derecho; BI: bronquio intermedio; BPI: bronquio principal izquierdo; EE: error estándar; DE: desviación estándar. 


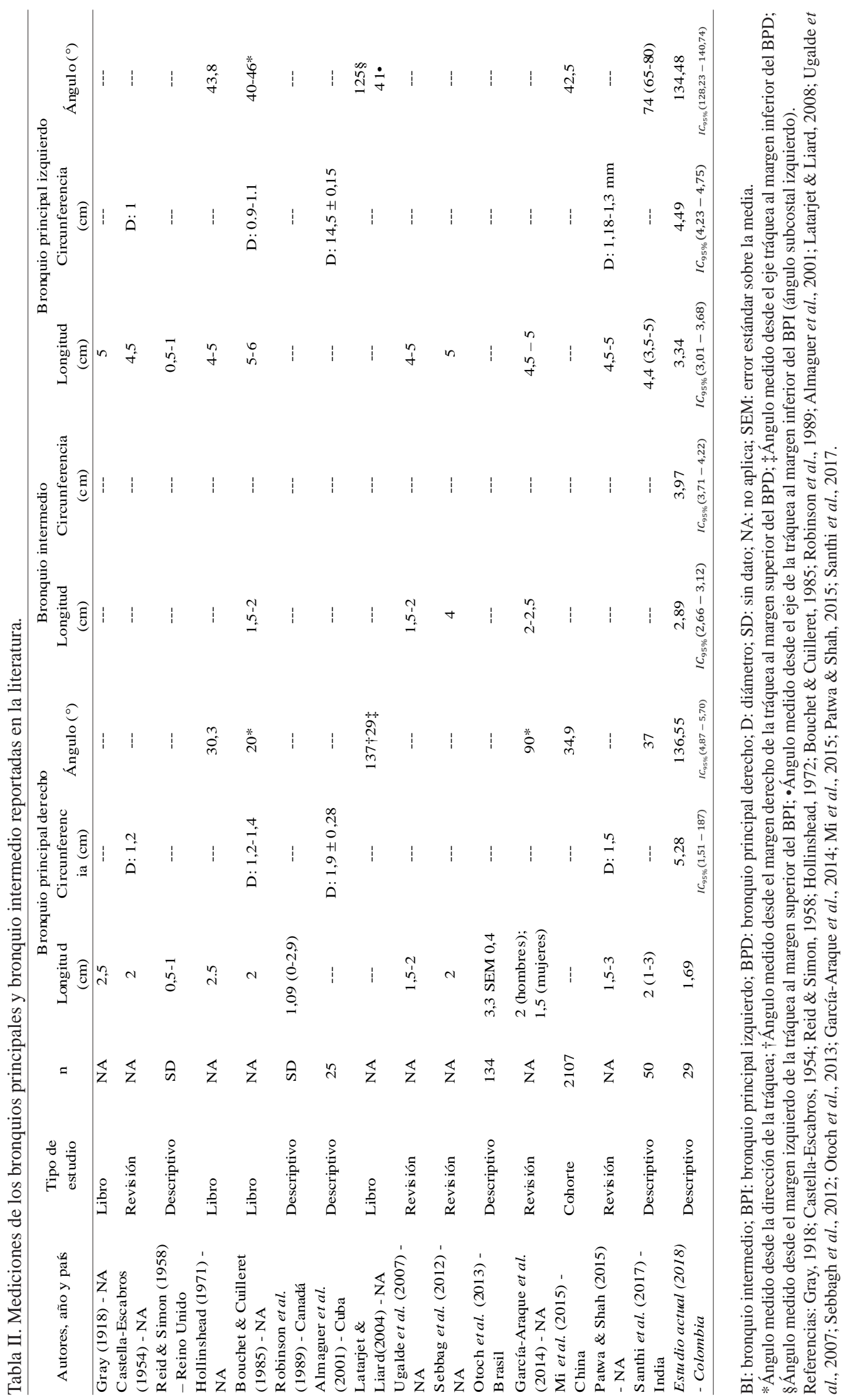

\section{DISCUSIÓN}

Este estudio presenta la morfometría de los bronquios principales y del BI en una muestra cadavérica, siendo la única serie reportada en la literatura hasta la fecha en Colombia. Diversos autores han descrito estas mediciones, principalmente como revisiones y textos de anatomía; sin embargo, los estudios realizados directamente con cadáver siguen siendo limitados, por lo que los valores aportados en el presente estudio resultan ser un punto de referencia en la investigación anatómica de los bronquios.

En la Tabla II se presentan las mediciones reportadas en la literatura. Los resultados obtenidos en este estudio sobre la longitud del BPD son semejantes a los reportados por Santhi (n=50, India), Robinson $(\mathrm{n}=24$, Canadá) y Otoch $(\mathrm{n}=100, \quad$ Brasil) (Robinson et al., 1989; Otoch et al., 2013; Santhi et al., 2017). Por otro lado, los autores del presente estudio encontraron que la circunferencia del BPD desde el origen tiene 
un promedio de $5,28 \mathrm{~cm}$, lo que concuerda con los datos obtenidos por Almaguer Rodríguez et al. en Cuba. Esta última medición debe considerarse en eventos que amenazan la vida como la broncoaspiración.

Respecto a la longitud del BPI, los hallazgos reportados en este estudio son menores que los presentados en textos de anatomía (Gray, 1918; Bouchet \& Cuilleret, 1985) y en el estudio descriptivo de Santhi et al., mientras que la circunferencia del BPI evidenció valores por encima de lo reportado por otros autores (Tabla II). En relación con la medición de ángulos, se encontró que los valores obtenidos en el presente estudio con respecto al ángulo interbronquial y al ángulo medido entre la tráquea y el BPI evidenciaron valores superiores a los descritos por Latarjet \& Ruiz Liard (2008), sin embargo, hubo semejanzas en ambos estudios con respecto a los valores obtenidos en la medición del ángulo entre la tráquea y el BPD. Llama la atención la poca evidencia disponible acerca de la medida de los ángulos formados entre la tráquea y los bronquios principales, pudiendo ser este un dato relevante en procedimientos imagenológicos e invasivos, tales como la broncoscopia, cirugía de tórax, toma de biopsias, interpretación de escanografías, entre otras.

Respecto al BI, no se encontraron estudios morfométricos donde se reporten mediciones de este, sino textos de anatomía o artículos de revisión que proveen dicha información sin un soporte metodológico; sin embargo, basados en los valores provistos por algunos textos, no se encontró una relación entre estos y los obtenidos en el presente estudio (Bouchet \& Cuilleret; Ugalde et al., 2007; Sebbagh et al.; García Araque et al.). Por otro lado, no se encontró información acerca de mediciones sobre el calibre del BI, por lo que este estudio es el primer trabajo realizado en cadáver que lo reporta.

A pesar de que existen múltiples discusiones y sistemas de clasificación realizadas el siglo pasado para la anatomía del árbol bronquial, no existen en las diferentes nomenclaturas argumentos claros acerca de la inclusión o exclusión del término BI. Por un lado, la nomenclatura de losbronquios segmentarios propuestos por el Comité Internacional en 1949 resolvió omitir el término de BI y reemplazarlo por el de parte inferior del BPD (Thoracic Society). Por otro lado, Minnich \& Mathisen definen al BI como una continuación del BPD que da lugar al BLM y al BLI. Pró (2012) por su parte, designa como BI al sector del BPD comprendido entre el margen inferior del origen del BLS y el margen superior del origen del BLM, su relación característica corresponde a que su cara anterior se encuentra cruzada de medial a lateral por la arteria pulmonar derecha, para situarse en el fondo de la fisura en la cara lateral del bronquio, ocupando la distancia entre los dos orígenes bronquiales precedentes. En el área de la radiología e imágenes diagnósticas, específicamente en el uso de escanografía, Hennings et al. (2002) ubican al BI inmediatamente posterior a la arteria pulmonar derecha y a la arteria pulmonar interlobar derecha.

Es por ello, que los autores del presente estudio proponen que el término de BI sea tenido en cuenta como una estructura separada de la denominación del BPD y de los bronquios lobares, con sus propias relaciones anatómicas y descripción, como la estructura bronquial que se origina a partir del BPD, que comprende el espacio entre el margen inferior del BLS y el margen superior del BLM, tal como fue descrito por Ewart, Latarjet \& Ruiz Liard y Pró; considerando como una relación anatómica característica del BI al cruce de la arteria pulmonar derecha de medial a lateral en su cara anterior. Por otro lado, teniendo en cuenta la escasa literatura acerca del tema y de mediciones en cadáver, se sugiere que las mediciones planteadas en el presente estudio sean consideradas como modelo estándar en cadáver (Tabla I).

Finalmente, dada la importancia de la localización del BI y sus relaciones anatómicas, se recomienda considerar que su definición sea incluida en la Nómina Anatómica con el fin de unificar conceptos y definiciones acerca de la organización del árbol bronquial y su anatomía.

\section{AGRADECIMIENTOS}

Los autores agradecen al Grupo de Tejidos Blandos y Mineralizados (TEBLAMI), perteneciente al Departamento de Morfología de la Universidad del Valle por su apoyo y colaboración. A Fabián Cabrera Pazmino por su colaboración en el diseño de las figuras del presente estudio.

PARRA-LARA, L. G.; CORRALES, L. M.; ZÚÑIGA, J. \& BAENA-CALDAS, G. P. Morphometric study of the main bronchi and the intermediate bronchus in a Colombian cadaveric sample. Int. J. Morphol., 37(4):1252-1257, 2019.

SUMMARY: Knowing morphometry in length, circumference and angulation of the main bronchi and the intermediate bronchus can be relevant data in radiological, surgical procedures and even in life-threatening events such as bronchoaspiration. Evidence is scarce about the morphometry of the main bronchi and the intermediate bronchus, that is why the present study aimed to describe the morphometry of the main bronchi and the intermediate bronchus in a Colombian cadaveric population sample. A descriptive study was carried out where 29 specimens were 
selected from Cali, Colombia; 11 corresponded to cadavers and 18 to organs isolated from the lower respiratory tract; cadavers with duly dissected structures were included and samples with anatomical structures that were absent or damaged, and that affected the performance of the measurements were excluded. Descriptive statistics was used to analyze the data. The mean length of the right main bronchus was $1.69 \mathrm{~cm} 95 \% \mathrm{CI}$ (1.51-1.87), the mean length of the intermediate bronchus was $2.89 \mathrm{~cm} 95 \%$ CI $(2,66-$ 3,12 ), the mean length of the left main bronchus was $3.34 \mathrm{~cm} 95 \%$ CI (3-3.68); the interbronchial angle had an average of $80.17^{\circ} 95$ $\%$ CI (72.87-87.48). The measurements of the right main bronchus agree with those reported in the literature, the opposite occurs with the left main bronchus; interbronchial angle measurement showed differences to that reported by the literature. This study shows that although there are international nomenclatures and consensus, these have not been implemented correctly by health professionals and the term intermediate bronchus is used in daily practice, it is therefore necessary that the Societies of Anatomy and Morphology again discuss their importance and recognition in the nomenclature.

\section{KEY WORDS: Anatomy; Bronchi; Cadaver.}

\section{REFERENCIAS BIBLIOGRÁFICAS}

Almaguer Rodríguez, C.; Durán Matos, M. \& Oliveros Viamontes, G. Diámetros del bronquio principal y vasos pulmonares a nivel del hilio del pulmón en el hombre. A. M. C., 5(2), 2001. Disponible en: http:// scielo.sld.cu/scielo.php?script $=$ sci_arttext\&pid $=$ S102502552001000200004

Alvarado-León, U.; Palacios-Acosta, J. M.; León-Hernández, A.; ChávezEnríquez, E. P.; Vásquez-Gutiérrez, V. M. \& Shalkow-Klincovstein, J. Cuerpos extraños alojados en las vías aéreas y digestiva. Diagnóstico y tratamiento. Acta Pediatr. Mex., 32(2):93-100, 2011.

Baharloo, F.; Veyckemans, F.; Francis, C.; Biettlot, M. P. \& Rodenstein, D. O. Tracheobronchial foreign bodies: presentation and management in children and adults. Chest, 115(5):1357-62, 1999.

Bouchet, A. \& Cuilleret, J. Anatomía Descriptiva, Topográfica y Funcional. Buenos Aires, Médica Panamericana, 1985.

Boyden, E. A. A critique of the international nomenclature on bronchopulmonary segments. Dis. Chest., 23(3):266-9, 1953.

Brock, B. The Anatomy of the Bronchial Tree: With Special Reference to the Surgery of Lung Access. New York, Oxford University Press, 1946.

Castella Escabros, A. Bronquios y segmentos pulmonares. An. Med. Cir., 34(111-112):297-315, 1954.

Comité Federal sobre Terminología Anatómica, International Federation of Associations of Anatomists \& Sociedad Anatómica Española. Terminología Anatómica: Terminología Anatómica Internacional. Madrid, Médica Panamericana, 2001.

Cortese, D. \& Prakash, U. Anatomy for the Bronchoscopist. New York, Raven Press, 1994.

Delgado García, A. Anatomía Humana Funcional y Clínica. Cali, Universidad del Valle, 1996.

Ewart, W. The Bronchi and Pulmonary Blood-Vessels. London, Churchill, 1889.

García Araque, H. F.; Valencia Orgaz, O.; López Vicente, R. \& Gutiérrez Vidal, S. E. Anatomía de la vía aérea para el broncoscopista. Una aproximación a la anestesia. Rev. Colomb. Anestesiol., 42(3):192-8, 2014.

Gray, H. Anatomy of the Human Body. $20^{\text {th }}$ ed. Philadelphia, Lea \& Febiger, 1918.
Hennings, H. E.; Zapata, D. M.; Vásquez, G. C.; Quiroz, R. L.; Samiento, A. M. \& Criales, C. J. L. Anatomía normal de la vía aérea y la segmentación pulmonar por tomografía computada helicoidal. An. Radiol. Mex., 1(4):587-9, 2002.

Hollinshead, W. H. Anatomy for Surgeons. Vol. 2: The Thorax, Abdomen $\&$ Pelvis. $2^{\text {nd }}$ ed. New York, Joanna Cotler Books, 1971.

Ikeda, S. Atlas of Flexible Bronchofiberscopy. Baltimore, University Park Press, 1974.

Jackson, C. L. \& Huber, J. F. Correlated applied anatomy of the bronchial tree and lungs with a system of nomenclature. Dis. Chest., 9(4):31926, 1943.

Latarjet, M. \& Ruiz Liard, A. Anatomía Humana. Buenos Aires, Médica Panamericana, 2008.

Mi, W.; Zhang, C.; Wang, H.; Cao, J.; Li, C.; Yang, L.; Guo, F.; Wang, X. \& Yang, T. Measurement and analysis of the tracheobronchial tree in Chinese population using computed tomography. PloS One, 10(4):e0123177, 2015.

Minnich, D. J. \& Mathisen, D. J. Anatomy of the trachea, carina, and bronchi. Thorac. Surg. Clin., 17(4):571-85, 2007.

Otoch, J. P.; Minamoto, H.; Perini, M.; Carneiro F. O. \& Artifon E. L. A. Is there a correlation between right bronchus length and diameter with age? J. Thorac. Dis., 5(3):306-9, 2013.

Patwa, A. \& Shah, A. Anatomy and physiology of respiratory system relevant to anaesthesia. Indian J. Anaesth., 59(9):533-41, 2015.

Pró, E. A. Anatomía Clínica. Buenos Aires, Médica Panamericana, 2012.

Reid, L. \& Simon, G. The peripheral pattern in the normal bronchogram and its relation to peripheral pulmonary anatomy. Thorax, 13(2):1039, 1958.

Robinson, C. L.; Müller, N. L. \& Essery, C. Clinical significance and measurement of the length of the right main bronchus. Can. J. Surg., 32(1):27-8, 1989.

Santhi, B.; Dhanalakshmi, V. \& Esther Yamuna, N. Trachea and mode of branching of bronchial tree: anatomical study. Int. J. Anat. Res., 5(3.2):4280-3, 2017.

Sebbagh, P. E.; Mordojovich, R. G. \& Undurraga, M. F. Anatomía radiológica del tórax. Rev. Chil. Enferm. Respir., 28(2):109-37, 2012.

Thoracic Society. The Nomenclature of Broncho-pulmonary Anatomy. An International Nomenclature Accepted by the Thoracic Society. Thorax, 5(3):222-8, 1950.

Ugalde, P.; Camargo, J. de J. \& Deslauriers, J. Lobes, fissures, and bronchopulmonary segments. Thorac. Surg. Clin., 17(4):587-99, 2007.

Dirección para correspondencia:

Gloria Patricia Baena-Caldas

Departamento de Morfología

Escuela de Ciencias Básicas

Facultad de Salud

Universidad del Valle

Calle 4B \# 36-00 Edificio 116

Cali

COLOMBIA

Email: gloria.baena@correounivalle.edu.co gpbaena@hotmail.com

Recibido : 08-02-2019

Aceptado: 10-06-2019 